\title{
Brucellosis hip abscess without risk factor
}

\author{
Elyazid Mouhsine $\cdot$ Alain Akiki $\cdot$ Raffaele Garofalo
}

Received: 13 August 2008/Accepted: 4 February 2009/Published online: 7 May 2009

(C) Springer-Verlag 2009

\begin{abstract}
Brucellosis has become a rare entity in many industrialised countries, because of animal vaccination programs. We report a first case in the literature of Brucella abscess in the hip region observed in Switzerland in a subject without any clear risk factor, leading us to conclude that abscess formation can be a rare manifestation of brucellosis. Because it can present in many different forms and locations without having characteristic clinics, a high index of suspicion is needed for the diagnosis even if the patient is a healthy athlete with no clear way of obvious route for contamination, and this even more if all the common causes of athletic hip pain have been ruled out.
\end{abstract}

Keywords Brucellosis · Joint $\cdot$ Hip $\cdot$ Malta fever

\section{Introduction}

Brucellosis, a zoonotic infection, transmitted to humans by lower animals, may present as a non-specific influenza-like febrile illnesses. Fever, headache, myalgia, arthralgia, back pain, chills and generalised weakness are the common signs.

In terms of musculoskeletal complications, the hip and the knee are the second most commonly affected joints after the sacroiliac joint [1-3]. The hip involvement in terms of arthritis has been widely described in literature, particularly in risk population [1]. However, abscess development is usually related to a direct causative factor [1]. This paper aims at presenting the first case in the

E. Mouhsine $(\bowtie) \cdot$ A. Akiki $~ R$ R. Garofalo

Department of Musculoskeletal Medicine, University Hospital,

OTR-BH 14, CHUV, 1011 Lausanne, Switzerland

e-mail: Elyazid.Mouhsine@hospvd.ch literature of Brucella abscess at the hip region in a subject without any clear risk factor.

\section{Case report}

A 32-year-old white hockey player presented to our consultation complaining of invalidating pain in the anterior region of his right hip; that started 10 days before without any previous notion of trauma. Anamnestically, the patient is known healthy, practises hockey regularly (3 times a week) and works as a financial consultant in a company. No associated fever was seen. Pain resolved within 15 days after the administration of anti-inflammatory drugs (Voltarene/diclofenac $50 \mathrm{mg}, 3$ times daily), combined with analgesic drugs (paracetamol $1 \mathrm{~g}, 4$ times daily) since a muscular tear was suspected.

After 6 months, the invalidating pain recurred even more intensely localised this time in groin region. Femoral hernia or irradiating sciatica was clinically excluded. $\mathrm{X}$-ray, magnetic resonance imaging (MRI) and bone scintigraphy were done to elucidate the cause of that continuing pain, but were all judged normal. In an attempt to resolve the unremitting pain, the patient was treated with oral steroids hydrocortone (hydrocortisone) $10 \mathrm{mg} 2$ times/day for 15 days), but without success. At this point, the diagnosis of "pubalgia" was retained and an infiltration of the symphysis pubis with local steroids was performed. After 1 week, the patient was readmitted to the hospital because of excruciating right groin pain accompanied with an acute deterioration of his general health with fever $\left(38.5^{\circ} \mathrm{C}\right)$, chills and arthralgia. Laboratory results showed a leucocytosis of $13.0 \mathrm{~g} / \mathrm{l}$ and a C-reactive protein (CRP) of $128 \mathrm{mg} / \mathrm{l}$. Hemocultures for common bacteria were negative. 
Fig. 1 X-ray of the right hip judged as normal
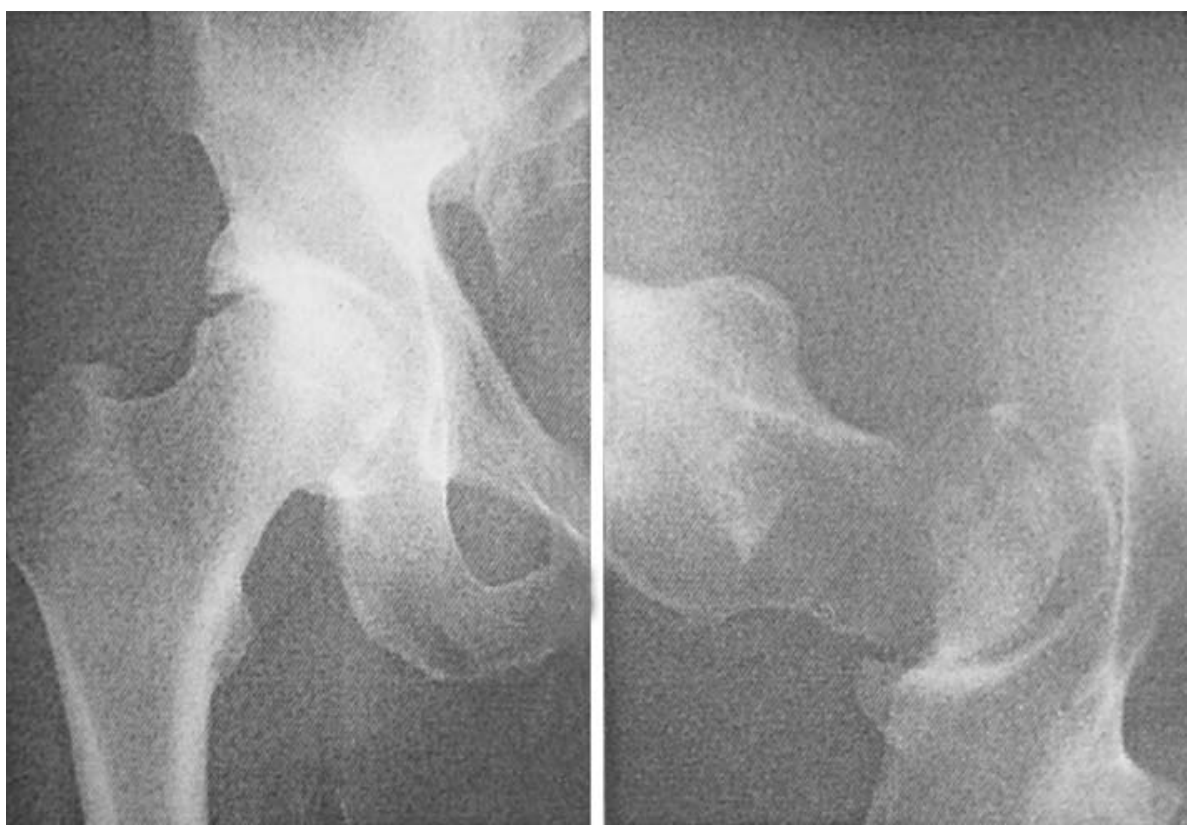

Right hip joint X-ray was normal (Fig. 1), but a new MRI (Fig. 2) revealed a high density mass at the level of the right acetabular roof and the gluteus minimus muscle. A computed tomography (CT)-guided punction was done and a purulent liquid was drained from the collection site. The CT scan control showed a net diminution of the collection volume. Two days later, the patient was pain free, independent and was discharged home. Antibiotic treatment combining oral rifampicin and doxycycline was instaured. This treatment was chosen because the preliminary results of the drained liquid revealed the presence of a gram-negative infection.

Ten days after his discharge, the patient had to be readmitted to the hospital for a recurrence of invalidating pain. In the mean time, serological results of the previously drained collection revealed the presence of Brucella bengalle. The treatment was now surgical and consisted of an arthrotomy, drainage and sequestrectomy of the right acetabulum combined with intravenous gentamycin (120 mg 2 times/day) and vibramycin (doxycycline) $100 \mathrm{mg} 2$ times/ day. The drainage of the abscess was done through a postero-lateral hip approach. During the surgery, an important remodelling of the posterior part of the acetabular roof was noted with infectious destruction that needed to be curetted. Local antibiotics (gentamicin) were placed deep in the right hip and closure was performed over two intraarticular suction drains.

Postoperatively, the patient had to follow bed rest for 6 weeks, with a $4 \mathrm{~kg}$ right-sided traction in order to protect the right acetabular roof. After this period, rehabilitation therapy was started and included partial weight-bearing
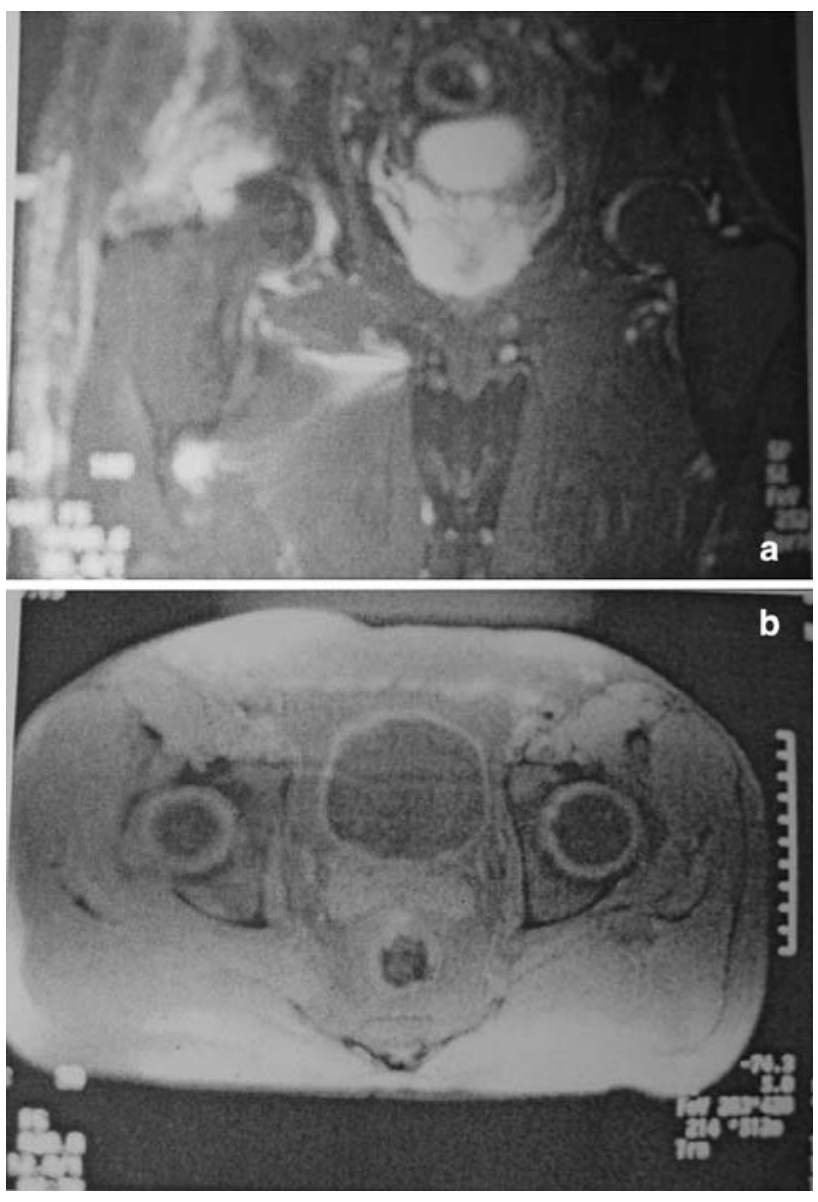

Fig. 2 a MRI showing a small collection at the upper border of the right acetabulum. $\mathbf{b}$ MRI showing that the infiltration interests also the right gluteus minimus muscle 
$(15-20 \mathrm{~kg})$ on the right side with the help of crutches for 6 weeks. Continuous passive and active mobilisation of the right hip was started in parallel. The patient was discharged 3 weeks later. After the 6 weeks control and a normal right-sided hip radiography, weight-bearing was increased progressively on the right hip allowing the patient to become independent at 3 months interval. The intravenous antibiotic treatment was continued ambulatory through a subcutaneous catheter for a total of 14 weeks. CRP results normalised and no further pain was felt. Complete healing and regain of function occurred 3 months later. Four months after surgery, work and sports activities could be resumed.

The patient was reviewed at one and followed up for 4 years. Clinically, he was pain free with a normal range of hip motion. On the X-ray, no changes of the articular area were visible (Fig. 3). Patient gave his written informed consent to the publication of his case report.

\section{Discussion}

Although brucellosis also known as undulant fever, Mediterranean or Malta fever has become a rare entity in many industrialised countries because of animal vaccination programs; this condition remains a significant health problem in many developing countries principally and, in particular, in the Mediterranean region, the Indian subcontinent and parts of Central and South America.

Brucellosis can occur at any age, but the most common age groups affected are adolescents and young adults [1,3]. Brucellosis with or without musculoskeletal involvement presents with non-specific clinical manifestations, such as

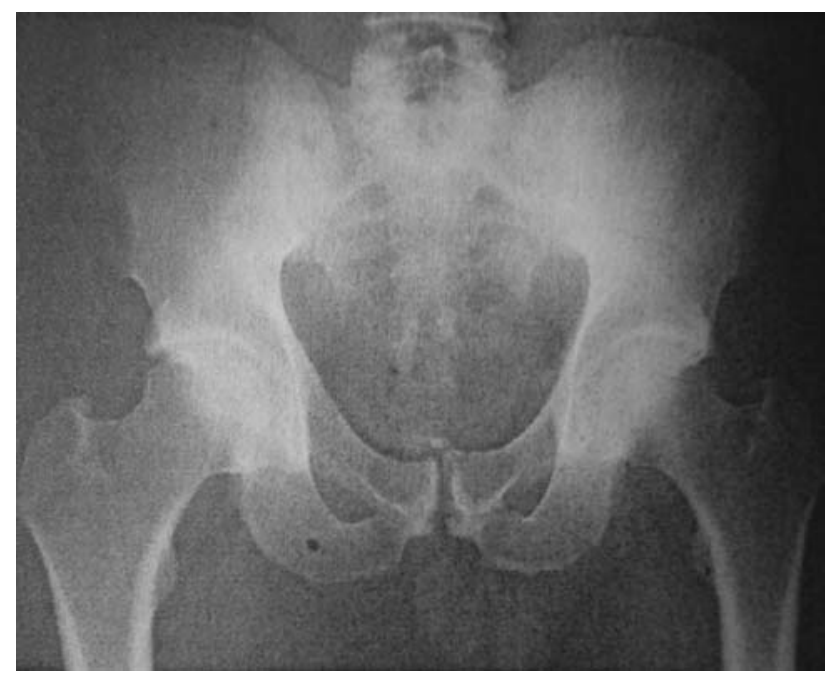

Fig. 3 Normal right hip X-ray 4 years after abscess drainage and traction fever, malaise, sweating, hepato or splenomegaly [1, 2, 4]. Routine laboratory data reported in most studies [5] are of little diagnostic help.

Complications occur with a certain predilection to the musculoskeletal system with a prevalence varying from 0 to $70 \%$ according to the stage of the disease and the rapidity of its diagnosis and treatment [4-8].

The prevalence and pattern of musculoskeletal involvement depend on the strain of Brucella, the patient's age and the duration of the disease [8].

Throughout the literature, the sacroiliac joint is the most affected part (55\%) $[5,8,9]$, but some authors report that in case of peripheral mono-arthritis, the hip and knee joints infections are predominant $[5,10,11]$.

The diagnosis of brucellosis depends primarily on the clinician's suspecting the presence of the disease because of the history and symptoms of the patient. Occupational history plays a crucial role, since individuals working in meat processing and livestock industries have accounted for more than $70 \%$ of reported cases [12]. In our case, the delay in diagnosis was mainly due to the absence of any helpful anamnestic information and the exact route of infection was unknown. The fact of the patient being an athlete, associated with an absence of any primary objective clinical or radiological explanation to his continuing pain contributed to the misleading process. Multiple structures in the hip have the potential to produce similar painful syndromes. The differential diagnosis of inguinal or hip pain in the athletic patient is wide and includes muscle tears (in particular adductors), abdominal muscular abnormalities, acetabular labral tears, inflammatory arthritis, osteitis pubis or hernias (femoral or inguinal). The character and localisation of pain is crucial to elicit the true cause of hip pain.

Nevertheless, the diagnosis of hip or groin pain remains challenging and the idea of an atypical adjacent infection should be kept in mind, especially in the presence of a chronic mono-articular joint affection. Because the clinical signs and symptoms of Brucella overlap with a variety of infectious and non-infectious diseases, the diagnosis can be perplexing. Localised abscess formation induced by Brucella is rare [8] but still an expected pathology, especially when an isolation of Brucella strain is possible from the culture. The majority of cases presenting as hip or groin pain associated with fever were attributed to infectious hip arthritis. In our case, however, we think that the primary process was the abscess formation in the gluteus minimus muscle. The delayed diagnosis is on the origin of the accompanying infection of the adjacent acetabular roof and the continuous manifestation of the symptomatology. To our knowledge, this is the first case of abscess Brucella of the hip region where usually all the feverish symptomatology is attributed to infectious hip arthritis. 
If an abscess is confirmed, the gold-standard remains the surgical drainage, decortication and adequate antibiotic therapy.

In conclusion, abscess formation is a rare manifestation of brucellosis. Because it can present in different forms and locations without having characteristic clinics, a high index of suspicion is needed for the diagnosis even if the patient is a healthy athlete with no clear way of contamination; and this even more if all the common causes of athletic hip pain have been excluded.

Conflict of interest statement The authors declare that they have no conflict of interest related to the publication of this manuscript.

\section{References}

1. Malik GM (1997) A clinical study of brucellosis in adults in the Asir region of southern Saudi Arabia. Am J Trop Med Hyg 56(4):375-377

2. Young EJ (2000) Brucella species. In: Mandell GL, Bennet JE, Dolin J (eds) Mandell, Douglas and Bennet's principles and practice of infectious disease, 5th edn. Churchill Livingstone, New York, pp 2386-2393
3. Malik GM (1997) A clinical study of brucellosis in adults in the Asir region of southern Saudi Arabia. Am J Trop Med Hyg 56(4):375-377

4. Lifeso RM, Harder E, McCorkell SJ (1985) Spinal brucellosis. J Bone Joint Surg 67:345-351

5. Colmenero JD, Reguera JM, Martos F, Sanchez-De-Mora D, Delgado M, Causse M, Martin-Farfan A, Juarez C (1996) Complications associated with Brucella melitensis infection: a study of 530 cases. Medicine (Baltimore) 75(4):195-211

6. Al-Rawi ZS, Al-Khateeb N, Khalifa SJ (1987) Brucella arthritis among Iraqi patients. Br J Rheumatol 26(1):24-27

7. Bocanegra TS, Gotuzzo E, Castaneda O, Alarcon GS, Espinoza LR (1986) Rheumatic manifestations of brucellosis. Ann Rheum Dis 45(6):526

8. Mousa ARM, Muhatseb SA, Almudallah DS et al (1987) Osteoarticular complications of brucellosis: a study of 169 cases. Rev Infect Dis 9:531-543

9. Tasova Y, Saltoglu N, Sahin G, Aksu HS (1999) Osteoarticular involvement of brucellosis in Turkey. Clin Rheumatol 18(3):214219

10. Fox MD, Kaufmann AF (1977) Brucellosis in the United States, 1965-1974. J Infect Dis 136:312-316

11. Joffee B, Diamond MT (1966) Brucellosis due to self-inoculation. Ann Intern Med 65(3):564-565

12. Trunnell TN, Waisman M, Trunnell TL (1985) Contact dermatitis caused by Brucella. Cutis 35(4):379-381 